\title{
Design of Grid Systems to Solve Complex Industrial Engineering Problems
}

\author{
Tynchenko Valeriya Valerievna \\ Dept. of Informatics \\ Siberian Federal University \\ Krasnoyarsk, Russia
}

\author{
Solovyov Evgeniy Alekseevich \\ Dept. of Production Machinery and Equipment for \\ Petroleum and Natural Gas Engineering \\ Siberian Federal University \\ Krasnoyarsk, Russia
}

\author{
Tynchenko Sergei Vasilyevich \\ Dept. of Economics and Information Technologies of management \\ Siberian Federal University \\ Krasnoyarsk, Russia
}

\begin{abstract}
The problem of solving complex and demanding tasks of production engineering is discussed. The complexity of the methods used in the solution of such tasks requires significant computational resources. It is proposed to use the widespread personal computers, combined into a single computer network on the basis of Grid technology. However, the informal aspect is the choice of an effective structure of such a system. It is proposed the complex of mathematical models for assessing performance and reliability of Grid systems and the corresponding problem of selecting its effective structure. On the basis of the proposed models the decision support system for the Grid systems formation is developed. To solve optimization problems arising in the process of decision making is proposed the using of the multiobjective genetic algorithm. In the study of the proposed system efficiency was solved the practical problem of designing effective grid system configuration, focused on the complex task of network planning by means of artificial neural networks.
\end{abstract}

Keywords- grid; optimization; genetic algorithm; decision support; modelling

\section{INTRODUCTION}

Industrial engineering is that part of normal engineering activities associated with optimization of existing and emerging processes and systems. The application of industrial engineering is inextricably linked with the use of quantitative methods, working with the information, which circulates in the production system. These methods often have a complicated mathematical justification. [1]

Since the foundation in industrial engineering are quite complex mathematical methods, the possibility of its application in greatly determined by the level of available computing technology.

The widespread use of high-performance and affordable personal computers, the development and large-scale practical application of network information technologies of different levels and purposes - all this allows to speak about distributed computer networks as effective and considerably cheaper alternative to multiprocessor and multicomputer systems as a hardware realization of computing in solving complex and demanding tasks. However, the main problem of the widespread technologies of global computer networks is the inability of a universal and effectively uses remote computing resources, because initially the so-called Internet technologies focused on data access, not to the computational performance. [2]

To overcome the limitations existing in the field of solutions enables the rapid development and introduction of advanced network technology Grid, which is based on the idea of creating a geographically distributed computing infrastructure, uniting resources of various types with shared access to these resources within the virtual organizations composed of businesses and professionals that share these common resources [3]. Using this technology and filling it with specific content, you can implement a particular Grid system designed for the solution of a class of applied tasks [4, 5].

Automation of Grid-systems resources distribution and their coordination in the process of solving complex scientific and engineering problems requires the development and application of formal methods of modeling and optimization for efficient configuration of Grid-systems computing resources that realize main functions.

\section{THE MATHEMATICAL MODELS OF THE GRID SYSTEMS PERFORMANCE AND RELIABILITY}

The decision support system (DSS) "The effective configuration of the Grid-system" is proposed to automate the selection of effective performance, reliability and cost structure of Grid system developed. This system is based on the proposed complex mathematical models to evaluate the performance and reliability of the Grid systems [6].

Formulation of the optimization problem of Grid systems effective configuration choice for solving complex problems of a certain class is as follows (1), (2): 


$$
\begin{aligned}
& \frac{\sum_{i=1}^{N_{T}-1} P\left(t_{i}, n_{k}, \omega_{s r v}^{k}, \bar{z}\right) \cdot\left(t_{i+1}-t_{i}\right)}{t_{N_{T}}-t_{1}} \rightarrow \max _{\bar{z}} \\
& C_{k}^{s r v}+\sum_{i=1}^{N} C_{i}^{c l} \cdot z_{i} \cdot\left|t_{i}^{\text {off }}-t_{i}^{\text {on }}\right| \rightarrow \min _{\bar{z}},
\end{aligned}
$$

under the conditions (3), (4), (5):

$$
\begin{gathered}
\frac{\sum_{i=1}^{N_{T}-1} K_{r}\left(n_{k}, \lambda_{k}^{s r v}, \mu_{k}^{s r v}, \lambda_{k}^{h u b}, \mu_{k}^{h u b}, \bar{z}\right) \cdot\left(t_{i+1}-t_{i}\right)}{t_{N_{T}}-t_{1}} \geq K_{r}^{\text {lim }} \\
\min _{i=\left\{1, \ldots, N_{T}-1\right\}} P\left(t_{i}, n_{k}, \omega_{s r v}^{k}, \bar{z}\right) \geq P^{\lim \mid} \\
N^{-} \leq \sum_{i=1}^{N} z_{i} \leq N^{+} .
\end{gathered}
$$

where $P(\cdot)$ - productivity criteria of the Grid system for solving complex problems of a certain class [6];

$P^{l i m}$ - the minimum level of the Grid system productivity;

$K_{r}(\cdot)$ - the Grid system availability coefficient $[6,7]$;

$K_{r}^{l i m}$ - the permissible level of the Grid system availability coefficient;

$N$ - the number of Grid system's client;

$M$ - the number of server nodes that available to include into Grid system configuration;

$k$ - the number of server node, included into considered Grid system configuration $(k=\overline{1, M})$;

$n_{k}$ - the number of processors in $k$-th server node;

$\omega_{s r v}^{k}$ - the productivity of each processor in $k$-th server node (FLOPS);

$\lambda_{k}^{s r v}$ и $\mu_{k}^{s r v}$ - the failure rate and the recovery rate of processors in $k$-th server node;

$\lambda_{k}^{h u b}$ и $\mu_{k}^{h u b}$ - the failure rate and the recovery rate of hub in $k$-th server node;

$\bar{z}-$ the vector of client node inclusion to the considered Grid system:

$$
\bar{z}: z_{i}=\left\{\begin{array}{l}
1, \text { i-th client node is included } \\
\text { to the considered Grid configuration }, i=\overline{1, N} \\
0, \text { otherwise }
\end{array}\right.
$$

$t_{i}$ - the points of ascending set of Grid system resources on and off times, including start and end points of time interval, $i=\overline{1, N_{T}}$;

$N_{T}$ - the number of ascending set points of Grid system resources on and off times, including start and end points of time interval, $N_{T}=2 \sum_{j=1}^{N} z_{j}+2$;

$C_{k}^{s r v}$ - the daily cost of Grid system $k$-th server node rent;

$C_{i}^{c l}$ - the cos per hour of Grid system $i$-th client node, $i=\overline{1, N}$;

$N^{+}$и $N^{-}$- the maximum and the minimum possible number of Grid system client nodes.

\section{THE PROBLEM SOLVING METHODS}

There are number of classical methods for multiobjective optimization: the method of additive convolution [8], method of limits [8], targeted programming [9], and the minimax approach [10]. Classical methods for Pareto set generation combine all criteria into a single function-convolution, and performed several runs of the optimization process with different settings of this function to find the optimal Pareto set [11]. Classical methods of multicriteria optimization are not well suited for solving complex problems. First, to obtain the Pareto-optimal set it requires multiple independent optimization cycles, the approval of which can cause an excessive amount of computation. Secondly, some methods may be sensitive to the nature of the Pareto-optimal front shape. In addition, it may require some additional information that is difficult or impossible to obtain. [12]

The application of the evolutionary approach allows overcoming the disadvantages of classical methods of multicriteria optimization [13]. In addition, evolutionary algorithms provide a global view of the search space of solutions that allow avoiding local minima and obtaining a lot of alternative solutions for one cycle of optimization.

Genetic algorithms (GA) are a class of evolutionary search techniques (genetic algorithms, genetic programming, evolutionary strategies, etc.) that simulate the processes of natural evolution in the course of solving an optimization problem [14]. The processes of evolution occurring in GA, are based on the following principle: "every biological species purposefully develops and changes in order to best adapt to the environment". Evolutionary search methods have been successfully used for solving various optimization problems, including optimization problems of engineering [15].

Evolutionary algorithms are based on collective training process inside the population of individuals, each of which represents a search point in the space of valid solutions for this problem. The population is initialized randomly and then covers the best regions of the search space through random processes of selection (which for some algorithms is not 
random, but well-defined), mutation and recombination (which is not representing in some implementations). [16, 17]

Thus, as a method for solving this optimization problem in a software system is used multiobjective genetic algorithm, the effectiveness of which depends weakly on the properties of the optimization functions that can cope with solving complex problems of global optimization [18].

Among the many existing at the present time multi-criteria implementations of GA was chosen the algorithm FFGA.

Method FFGA [19] is based on Pareto-dominance ranking procedure of individuals, where the rank of each individual is determined by the number of individuals dominating it.

The problem of accounting restrictions in the system of decision support was solved by a method of adaptive penalties, where penalty functions depend not only on the number of iterations, but on the number of hits the best individuals at each step in a valid or invalid region. [20]

In this method there is a reduction of the fine to $(\mathrm{t}+1)$-th step if the best individual of the population (in terms of suitability) for the last $\mathrm{k}$ iterations belonged to the permissible range. If the best individual of the population during the same time period went beyond the bounds of the valid region, there is an increase of the fine.

\section{THE DECISION SUPPORT SYSTEM}

The decision support system "The effective configuration of the Grid-system" was invented in this paper, which generalize structure diagram consists of 2 modules and 6 subsystems (fig. 1).

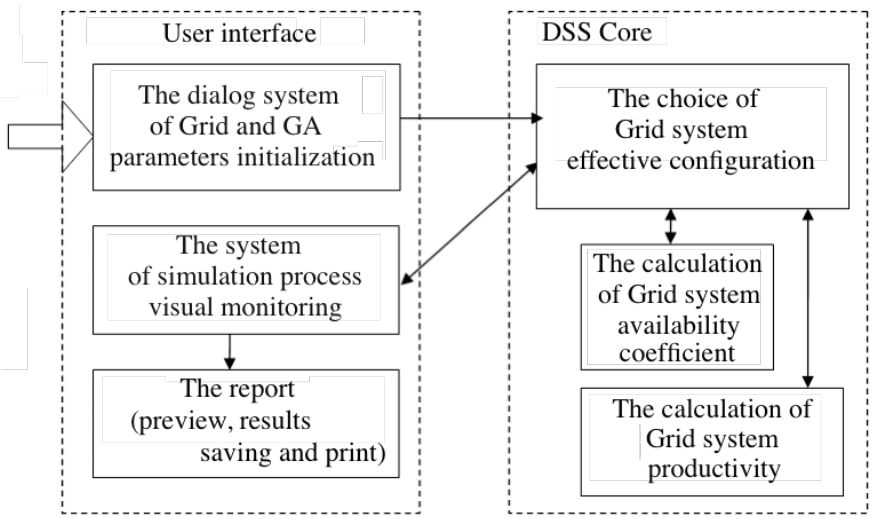

Fig. 1. The decision support system generalize structure diagram.

The following functionality implemented in this software system:

- configuration of hardware characteristics of Grid system's client and server nodes;

- customization of Grid system client nodes schedules;

- setting of algorithm parameters;

- setting GA parameters for Grid-system effective configuration choice;
- visualization of the information, that characterize a process of problem solving;

- changing GA settings in the process of problem solving;

- visualization of results according to the choice of Gridsystem effective configuration;

- saving results in text and graphical files.

The results of the software system:

- the set of non-dominated configurations of the designed Grid systems, each of which is characterized by the following information about its composition and characteristics: the hardware characteristics of the client nodes and servers, performance and cost of the system, the value of the availability coefficient;

- approximation of the Pareto-optimal front;

- fitness diagrams of the best and the worst individuals, as well as the average fitness of the GA population.

\section{THE PRACTICAL PROBLEM SOLUTION.}

The developed software system was used in the LLC "Regional information technology systems" (Krasnoyarsk, Russia) for the distributed solution of the problem of choosing the effective centralized Grid system structure. The designed system is tuned to the solution of computationally difficult problems of network planning with neural network modeling methods.

The characteristics of the tasks have been previously evaluated:

- the average computational difficulty of one iteration of calculations to the one branch of the algorithm - 10000 operations;

- the average computational difficulty of the one branch of the algorithm management -5000 operations;

- the average amount of client-server exchange data -200 KB.

For building Grid systems available 153 client node with a given schedule and the following parameters:

- productivity - from 4200 to 17345 MFLOPS;

- data transmission channels speed - from $10 \mathrm{Mbit} / \mathrm{s}$ to 27 $\mathrm{Mbit} / \mathrm{s}$;

- rental price per 1 hour of use - from $\$ 0.1$ to $\$ 0.38$;

- all client nodes are divided into 2 groups by their reliability - failure rate 0,00001 and 0,000001 .

The choice of server node was not made for the reason that the customer provides the server node for free with the following parameters:

- the number of processors - 2 ;

- the performance of each server CPU - 11500 MFLOPS;

- the rental price is $\$ 0$; 
- the failure rate of processors on the server - 0,000001.

The projected Grid system has the following restrictions:

- the number of client nodes Grid-system - from 15 to 20;

- availability coefficient of Grid-system is not less than $99.9 \%$;

- the minimum productivity of Grid-system is at least 50 GFLOPS.

As the result of the problem solution was obtained approximation of the Grid-system configurations Pareto-set, consisting of 6 points, as well as his front.

Approximation of the Pareto front presented in fig. 2.

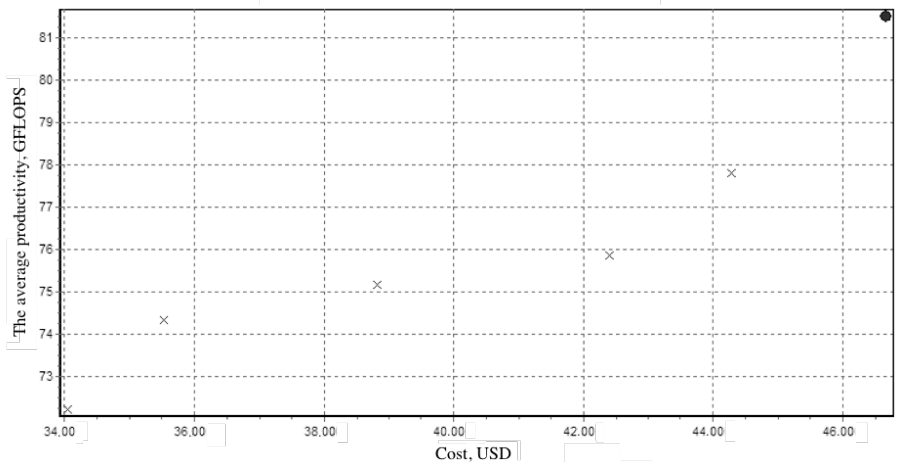

Fig. 2. The approximation of the Pareto front.

On the values of the cost and performance of obtained Grid systems configurations have been selected the following option:

- average performance - 81,522 GFLOPS;

- cost - \$46,67 per day;

- availability - 99,92\%;

- minimum performance - 51 GFLOPS.

\section{CONCLUSION}

Thus, with use of the developed DSS was chosen the effective configuration of the centralized type Grid-system, which allows implementing distributed computing for a given algorithm.

The results of the practical testing show that the developed software allows to successfully solve the problem of designing computing systems based on Grid technologies, destined to solve complex scientific and engineering problems.

\section{REFERENCES}

[1] Mauergauz, Yu.E. The advanced planning and scheduling in the production and supply chains / Yu. E. Mauergauz. - Moscow: Economics, 2012. - 576 p.

[2] Berman, F. Application-Level Scheduling on Distributed Heterogeneous Networks / F. Berman, R. Wolski, S. Figueira, J. Schopf, G. Shao // In Proc. Supercomputing '96, 1996. - P. 69-75.

[3] Foster, I. The Anatomy of the Grid: Enabling Scalable Virtual Organizations / I. Foster, C. Kesselman, S. Tuecke // International
Journal of High Performance Computing Applications. - 2001. - 15(3). - P. 200-222.

[4] Kiryanov, A.K. Introduction to the Grid technology / A.K. Kiryanov, Yu. F. Ryabov. - Gatchina : PIYAF RAN, 2006. - 39 p.

[5] Foster, I. The Physiology of the Grid: An Open Grid Services Architecture for Distributed Systems Integration. / I. Foster, C. Kesselman, J. M. Nick, S. Tuecke // URL: http://www.globus.org/research/papers/ogsa.pdf

[6] Efimov, S.N. Models and algorithms for GRID-systems design for neuronet model structure-parametric synthesys / S.N. Efimov, V.S. Tynchenko // Vestnik of Siberian State Aerospace University. - 2008. Vol. 4 (21). - P. 18-22.

[7] Pavsky, V.A. Calculation of computing systems reliability indicators / V.A. Pavsky, V.G. Horoshevsky // «Artificial intelligence. Intellectual and multiprocessor systems». - Taganrog : TRTU, 2006. - Vol.2. - P. 17-21.

[8] Cohon, J. Multiobjective Programming and Planing / J. Cohon. - New York : John Wiley, 1978. - 175 p.

[9] Steuer, R.E. Multiple Criteria Optimization / R.E. Steuer. - New York : John Wiley, 1986. - 267 p.

[10] Koski, J. Multi-criteria Desighn Optimization / J. Koski, A. Oscyczka. Springer-Verlag, 1990. - $154 \mathrm{p}$.

[11] Zitzler, E. Multiobjective evolutionary algorithms: A comparative case study and the strength Pareto approach / E. Zitzler, L. Thiele // IEEE Transactions on Evolutionary Computation. - 1999.

[12] Rastrigin, L.A. The stochastic search / L.A. Rastrigin. - Moscow : Znaniye, 1979. - $196 \mathrm{p}$.

[13] Booker, L. Improving search in genetic algorithms / L. Booker // L. Genetic algorithms and Simulated Annealing. - London : Pitman, 1987. - P. 61-73.

[14] De Jong, K. A Genetic Algorithms: A 10 Year Perspective / K. De Jong // The First Int. Conf. on Genetic Algorithms: Proceedings. - 1985. P.167-177.

[15] Rutkovskaya, D. Neural networks, genetic algorithms and fuzzy systems. M. : Hot Line - Telecom, 2004. - 452 p.

[16] Haupt, R.L. Practical Genetic Algorithms / R.L. Haupt, S.E. Haupt. 2ed. - Wiley, 2004. - 261p.

[17] Fourman, M. P. Compaction of symbolic layout using genetic algorithms / M. P. Fourman // The First International Conference on Genetic Algorithms and Their Applications : Proceedings. - Hillsdale, NJ : Lawrence Erlbaum, 1985. - P. 141-153.

[18] Zitzler, E. Multiobjective evolutionary algorithms: A comparative case study and the strength Pareto approach / E. Zitzler, L. Thiele // IEEE Transactions on Evolutionary Computation. - 1999.

[19] Fonseca, C. M. Multiobjective optimization and multiple constraint handling with evolutionary algorithms - Parts I, II: A unified formulation / C. M. Fonseca, P. J. Fleming // Technical report 564. - Sheffield, UK : University of Sheffield, 1995, January.

[20] Michalewicz, Z. Evolutionary algorithms for constrained optimization problems / Z. Michalewicz, M. Schoenauer // Evolutionary Computation, 4:1, pp. 1-32, 1996. 